Pure Appl. Chem., Vol. 81, No. 8, pp. 1397-1411, 2009.

doi:10.1351/PAC-CON-08-06-04

(C) 2009 IUPAC, Publication date (Web): 28 July 2009

\title{
Density functional theory study of the Jahn-Teller effect in cobaltocene*
}

\author{
Matija Zlatar ${ }^{1,2}$, Carl-Wilhelm Schläpfer ${ }^{1}$, Emmanuel Penka Fowe ${ }^{1}$, \\ and Claude Daul ${ }^{1, \ddagger}$
}

\author{
${ }^{1}$ Department of Chemistry, University of Fribourg, Fribourg, Switzerland; ${ }^{2}$ Center \\ for Chemistry, IHTM, University of Belgrade, Belgrade, Serbia
}

\begin{abstract}
A detailed discussion of the potential energy surface of bis(cyclopentadienyl)cobalt(II), cobaltocene, is given. Vibronic coupling coefficients are calculated using density functional theory (DFT). Results are in good agreement with experimental findings. On the basis of our calculation, there is no second-order Jahn-Teller (JT) effect as predicted by group theory. The JT distortion can be expressed as a linear combination of all totally symmetric normal modes of the low-symmetry, minimum-energy conformation. The out-ofplane ring deformation is the most important mode. The JT distortion is analyzed by seeking the path of minimal energy of the adiabatic potential energy surface.
\end{abstract}

Keywords: Jahn-Teller effect; DFT; cobaltocene; vibronic coupling; intrinsic reaction coordinate.

\section{INTRODUCTION}

The Jahn-Teller (JT) effect [1] is the subject of extensive research, both experimental and theoretical. The JT theorem states that a molecule with a degenerate electronic ground state will spontaneously distort along non-totally symmetric vibrational coordinates. This removes the degeneracy and lowers the energy. The theory underlying the JT and related effects is known and documented in detail [2-4]. The description of JT phenomena is based on a perturbation expression of the potential energy surface at and near the point of electronic degeneracy. Group theory allows identifying the symmetry of the distortion.

This effect is frequently observed in transition-metal chemistry [5,6], where degenerate ground states (e.g., in coordination compounds) are common. Density functional theory (DFT) is the modern alternative to the wavefunction-based ab initio methods. Although it is the most common theoretical method in quantum chemistry, up to now DFT is rarely used to discuss JT problems because of erroneous beliefs that it is not able to handle degenerate states [7]. Contrary to this, DFT can be applied to both degenerate and excited states, as formally proved by reformulation of the original Hohenberg-Kohn theorems - constrained search method and finite-temperature DFT [8]. Additionally, conventional single-determinant DFT has been extended to handle multiplet problem $[9,10]$. Application of multideterminental DFT to the JT systems has been developed in our group [11] and used with success [11-13].

\footnotetext{
*Paper based on a presentation at the $8^{\text {th }}$ Conference on Solid State Chemistry, 6-11 July 2008, Bratislava, Slovakia. Other presentations are published in this issue, pp. 1345-1534.

${ }^{\ddagger}$ Corresponding author
} 
In this paper, we show the power of our method for the analysis of the JT effect. As a particular example, we use bis(cyclopentadienyl)cobalt(II) (cobaltocene), a metallocene with a $d^{7}$ electronic configuration, which has been a matter of extensive research for years [14-21]. The distortion of cobaltocene is discussed qualitatively using group theory arguments, and the potential energy surface is calculated by a simple computational recipe [11], using vibronic coupling. The intrinsic reaction coordinate (IRC) method [22-25] is used to analyze the distortion vector which describes the direction and magnitude of the JT coordinate. The former is analyzed in terms of normal vibrations of the stable low-symmetry conformation. Thereupon, we propose the new approach to deal with the multimode problem [2], and to analyze it along the minimal energy path, from JT cusp to the geometry minima.

\section{RELEVANT THEORY OF THE JAHN-TELLER EFFECT}

Consider a N-atomic molecule in a high-symmetry nuclear configuration, $\vec{R}_{H}$, in the point-group $G_{0}$. The Hamiltonian $\hat{H}^{0}$ defines the electronic structure. Suppose that the ground state with energy $E^{0}$ is $f$-fold degenerate $\left(\Psi_{1}^{0}, \Psi_{2}^{0}, \ldots, \Psi_{f}^{0}\right)$. The molecule has $3 N-6$ normal coordinates $Q_{k}(3 N-5$ if it is linear), which describes all possible distortions. In order to discuss the potential energy surface, the electronic Hamiltonian, $\hat{H}$, is expanded as a Taylor series around the high-symmetry point $\vec{R}_{H}$ along the orthonormal $Q_{k}$ s:

$$
\hat{H}=\hat{H}^{0}+\sum_{k=1}^{3 N-6}\left(\frac{\partial V}{\partial Q_{k}}\right)_{0} Q_{k}+\frac{1}{2} \sum_{k, l=1}^{3 N-6}\left(\frac{\partial^{2} V}{\partial Q_{k} Q_{l}}\right)_{0} Q_{k} Q_{l}+\cdots=\hat{H}^{0}+\hat{W}
$$

$\hat{W}$ represents the vibronic operator (JT Hamiltonian) and is treated as a perturbation to $\hat{H}^{0}$. The subscript 0 indicates that analysis is performed in high symmetry. In order to calculate the potential energy surface, the following secular equation has to be solved:

$$
\left|\begin{array}{cccc}
H_{11}-E & H_{12} & \cdots & H_{1 f} \\
H_{21} & H_{22}-E & \cdots & H_{2 f} \\
\cdots & \cdots & \cdots & \cdots \\
H_{f 1} & H_{f 2} & \cdots & H_{f f}-E
\end{array}\right|=0
$$

$H_{i j}$ are the matrix elements of the Hamiltonian $\hat{H}$ in the basis of $\Psi_{1}^{0}, \Psi_{2}^{0}, \ldots, \Psi_{f}^{0}$. Using conventional second-order perturbation theory, they are given by eq. 3 :

$$
H_{i j}=E^{0} \delta_{i j}+\sum_{i, j=1}^{f}\left\langle\Psi_{i}^{0}|\hat{W}| \Psi_{j}^{0}\right\rangle+\sum_{i=1}^{f} \sum_{m \neq 0} \frac{\left|\left\langle\Psi_{j}^{0}|\hat{W}| \Psi^{m}\right\rangle\right|^{2}}{E^{0}-E^{m}}+\cdots
$$

This formulation allows a discussion of the JT effect [1], the pseudo-Jahn-Teller (PJT) effect [2,3], the Renner-Teller (RT) effect [2,26], as well as the chemical reactivity [3,27-29], with the same formalism [2]. 
Considering the terms up to second order in $Q_{k}$ leads to the following expression:

$$
\begin{aligned}
& H_{i j}=E^{0} \delta_{i j}+\sum_{k}^{3 N-6} \sum_{i, j=1}^{f}\left\langle\Psi_{i}^{0}\left|\left(\frac{\partial V}{\partial Q_{k}}\right)_{0}\right| \Psi_{j}^{0}\right\rangle Q_{k}+ \\
& +\frac{1}{2} \sum_{k, l=1}^{3 N-6} \sum_{i, j=1}^{f}\left\langle\Psi_{j}^{0}\left|\left(\frac{\partial^{2} V}{\partial Q_{k} \partial Q_{l}}\right)_{0}\right| \Psi_{i}^{0}\right\rangle Q_{k} Q_{l}+\sum_{k=1}^{3 N-6} \sum_{i=1}^{f} \sum_{m \neq 0} \frac{\left|\left\langle\Psi_{i}^{0}\left|\left(\frac{\partial V}{\partial Q_{k}}\right)_{0}\right| \Psi^{m}\right\rangle\right|^{2}}{E^{0}-E^{m}} Q_{k}^{2}
\end{aligned}
$$

The matrix elements in eq. 4 are called vibronic coupling constants, and some of them have a clear physical meaning [2]: $F_{i j}^{k}=\left\langle\Psi_{i}^{0}\left|\left(\frac{\partial V}{\partial Q_{k}}\right)_{0}\right| \Psi_{j}^{0}\right\rangle$ are the linear vibronic coupling constants; $G_{i j}^{k l}=\left\langle\Psi_{i}^{0}\left|\left(\frac{\partial^{2} V}{\partial Q_{k} \partial Q_{l}}\right)_{0}\right| \Psi_{j}^{0}\right\rangle$ are the quadratic vibronic coupling constants; $G_{i i}^{k k}=\left\langle\Psi_{i}^{0}\left(\frac{\partial^{2} V}{\partial Q_{k}^{2}}\right)_{0} \Psi_{i}^{0}\right\rangle=K_{0}$ are the harmonic force constants; $R_{i m}=\frac{\left|\left\langle\Psi_{i}^{0}\left|\left(\frac{\partial V}{\partial Q_{k}}\right)_{0}\right| \Psi_{j}^{0}\right\rangle\right|^{2}}{E^{0}-E^{m}}=\frac{1}{2} K_{v}$ is the electronic relaxation.

The slope of the potential energy along the direction $Q_{k},\left(\partial E / \partial Q_{k}\right)_{0}$ is given by the diagonal linear vibronic constant, $F_{i i}^{k}$. These terms are zero at any extremal point. If the ground state is nondegenerate, the integral will vanish unless $Q_{k}$ is totally symmetric. Therefore, in a system with a nondegenerate ground state, the potential energy surface shows only a gradient along totally symmetric distortions. As a consequence, for any nonstationary point, the point group is conserved on the way to the minimum [3]. If the ground state is degenerate, however, $Q_{k}$ might be a basis for a non-totally symmetric representation. For this it must belong to the same representation as the direct product of the components of the degenerate ground state. The spontaneous distortion along these non-totally symmetric normal coordinates, $Q_{k}$ s, leads to a descent in symmetry and removes the degeneracy. Jahn and Teller [1] examined all degenerate terms of the symmetry point groups of nonlinear molecules and showed that there is always at least one non-totally symmetric vibration for which $F_{i j}^{k} \neq 0$. This is the physical basis of the (first-order) JT effect. The JT problems are classified according to the symmetry types of the electronic states and the vibrations that are coupled [2]. For example, the $\mathrm{E} \otimes e \mathrm{JT}$ problem denotes coupling of the degenerate electronic state of irrep E, by a degenerate vibration of irrep $e$. Since the slope of the potential surface at the high-symmetry configuration, $\vec{R}_{H}$, is nonzero, this point is not a stationary point. It represents a cusp of the potential energy surface obtained in conventional DFT.

Curvature of the potential energy surface in the direction $Q_{k}$ is measured with the force constant, $K_{k}=1 / 2\left(\partial^{2} E / \partial Q_{k}^{2}\right)=K_{0}+K_{v}$ [2]. The diagonal matrix elements of the second derivative of the potential energy operator, $G_{i i}^{k k}$, are primary or nonvibronic force constants, $K_{0}$ [2]. They are always different from zero and positive, representing a restoring force that tends to bring the system back to the more symmetrical situation.

Electronic relaxation, $R_{i m}$, depicts the coupling of the ground state $\Psi_{i}^{0}$ with excited states $\Psi^{m}$. This term is always negative. Generally, it is different from zero, as there will always be some excited states of proper symmetry that match those with a direct product of the representations of the ground 
state and the normal coordinate $Q_{k}$. It becomes increasingly important when the ground and the excited states are close in energy. The negative curvature of the potential energy surface at a transition state is a result of this term [3]. Therefore, it is referred to as the vibronic force constant, $K_{v}$. It is responsible for the pseudo-JT effect, configurational instability of polyatomic species with nondegenerate electronic states, for the avoided crossing between states of the same symmetry, and for the softening of the ground-state curvature at the minimum point, and it contributes to the anharmonicity of the vibrations [2,3,27-29]. In practice, in the analysis of JT systems, this term is usually neglected, or added to the total, observed force constant $K_{k}$.

The quadratic constants, $G_{i j}^{k l}$, in nonlinear molecules are usually not responsible for lowering the symmetry, but influence the shape of the potential energy surfaces, e.g., warping of the "Mexican hat" in the $\mathrm{E} \otimes e \mathrm{JT}$ problem [2]. For linear molecules, the linear vibronic constants are always zero because the non-totally symmetric vibrations are odd and degenerate states are even, but the quadratic terms are nonzero, and this may lead to instability of the linear configurations in case of sufficiently strong coupling. This is the physical basis of the RT effect $[2,26]$.

As we see, the complexity of eq. 4 is reduced by symmetry rules, which allow us to identify the nonzero vibronic coupling constants. One should note that matrix elements in eq. 4 contain all the components of the degenerate electronic state $(i, j, \ldots, f)$ and their combinations. The Wiegner-Eckart theorem $[30,31]$ states, that for any operator $\chi_{\Gamma_{k}^{p}}$ which transforms according to irreducible representation $\Gamma_{k}^{\prime \prime}\left(k\right.$ is a component of the degenerate representation) the matrix elements of the form $\left\langle\Gamma_{i}\left|\chi_{\Gamma_{k}^{\prime}}\right| \Gamma_{j}^{\prime}\right\rangle$ are given by the product of a reduced matrix element and coupling coefficients $C_{\Gamma{ }^{\prime \prime}}^{i j k} \Gamma^{\prime}$ of the point group $G_{0}$ :

$$
\left\langle\Gamma_{i}\left|\chi_{\Gamma_{k}^{\prime \prime}}\right| \Gamma_{j}^{\prime}\right\rangle=C_{\Gamma \Gamma \Gamma^{\prime}}^{i j k}\left\langle\Gamma\left\|\chi_{\Gamma^{\prime \prime}}\right\| \Gamma^{\prime}\right\rangle
$$

$\left\langle\Gamma|| \chi_{\Gamma^{\prime \prime}}|| \Gamma^{\prime}\right\rangle$, the reduced matrix element does not depend on the $i, j, k$. Thus, if the values of the coupling coefficients are known, only one reduced matrix element has to be calculated or determined experimentally. This simplifies the problem considerably. In the case of a $\mathrm{E} \otimes e \mathrm{JT}$ problem, the potential energy surface is determined by only three reduced matrix elements, corresponding to the parameters $F, G, K[2]$.

The point group $G_{1}$ of the extremal points on the potential energy surface, which is of lower symmetry than the point group $G_{0}$ of the degenerate configuration, can be predicted by the group theory using the epikernal principle $[2,32,33]$. The symmetry of stationary points is the highest possible one with lifted degeneracy, i.e., where the distortion coordinate becomes totally symmetric [3].

As a conclusion of this part, we observe that for molecules in high-symmetry configurations or for transition states of chemical reactions, JT vibronic coupling (JT, RT, and PJT effects) is the only possible source of spontaneous distortion, which leads to the breaking of symmetry in nature [2].

\section{JAHN-TELLER PROBLEM IN $D_{5}$ SYMMETRY}

The high-symmetry conformation of metallocenes can be either $D_{5 h}$ when the two rings are eclipsed or $D_{5 d}$ when the two rings are staggered. In both cases, the symmetry arguments are the same as for an intermediate structure of $D_{5}$ symmetry.

The ground electronic state of cobaltocene in $D_{5 h}$ symmetry is ${ }^{2} \mathrm{E}_{1} ",{ }^{2} \mathrm{E}_{1 \mathrm{~g}}$ in $D_{5 d}$, with a single electron in the doubly degenerate orbital. Thus, the system is subject to a first-order JT effect. Using group theory, it is easy to show that the distortion coordinate is $e_{2}$ in the eclipsed and $e_{2 g}$ in the staggered conformation, and the descent in symmetry goes to $C_{2 v}$ and $C_{2 h}$, respectively. The electronic states will split: $\mathrm{E}_{1}$ " into $\mathrm{A}_{2}$ and $\mathrm{B}_{1} ; \mathrm{E}_{1 \mathrm{~g}}$ into $\mathrm{A}_{\mathrm{g}}$ and $\mathrm{B}_{\mathrm{g}}$; the $\mathrm{JT}$ active distortion $e_{2}^{\prime}$ splits into $a_{1}$ and $b_{2} ; e_{2 g}$ into $a_{g}$ and $b_{g}$. For the sake of simplicity in the discussion, the symbols of $D_{5} \rightarrow C_{2}$ are used throughout. They are $\mathrm{E}_{1}$ for the ground state and $e_{2}$ for the JT active distortion. In $C_{2}$, the former splits 
into A, B, and later into a, b. This is schematically shown in Fig. 1. The results of the calculation are given for both conformations, $D_{5 h}$ and $D_{5 d}$.

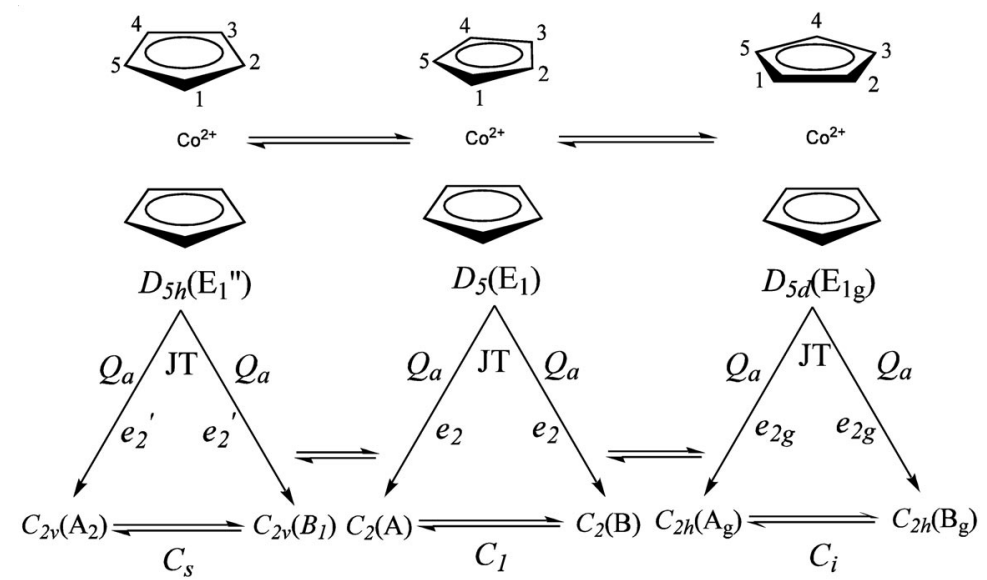

Fig. 1 Summary of the JT effect in cobaltocene. Symmetries of the corresponding geometries, electronic states and normal coordinates $\left(Q_{a}\right)$, as well as the numbering of $\mathrm{C}$ atoms in the cyclopentadienyl rings is given.

To get the potential energy surface, the $2 \times 2$ secular equation (eq. 2), expressed in the basis of the two degenerate components of the $\mathrm{E}_{1}$ ground state, $\Psi_{A}$, which belongs to the $\mathrm{A}$ in $C_{2}$ and $\Psi_{B}$ which belongs to $\mathrm{B}$ must be solved. The symmetry properties of the wavefunctions are the same as those of real $3 \mathrm{~d}_{y z}$ and $3 \mathrm{~d}_{x z}$ orbitals. The problem is analyzed in the space of the two components $Q_{a}$ and $Q_{b}$ of $e_{2}$. $Q_{a}$ is of $a$ symmetry in $C_{2}$, i.e., like the $3 \mathrm{~d}_{x 2-y 2}$ orbital, $Q_{b}$, is of $b$ symmetry in $C_{2}$, as is $3 \mathrm{~d}_{x y}$. The potential along the direction of the JT inactive vibrations is parabolic with a minimum for the high-symmetry conformation. The JT active distortion is the totally symmetric reaction coordinate, $a$, in $C_{2}$. The modes of $b$ symmetry allow mixing of the two electronic states emerging from the degenerate ground state.

Due to the properties of the $D_{5}$ group, the JT problem is simplified because $e_{2} \otimes e_{2} \subset a_{1}+$ $\left[a_{2}\right]+e_{1}$ and there are no terms of $e_{2}$ symmetry to interact with the $\mathrm{E}_{1}$ electronic wavefunctions. Therefore, the second-order vibronic coupling constant $G_{A B}^{a b}$ is zero.

$$
G_{A B}^{a b}=\left\langle\Psi_{A}^{0}\left|\left(\frac{\partial^{2} V}{\partial Q_{a} \partial Q_{b}}\right)\right| \Psi_{B}^{0}\right\rangle=\left\langle\Psi_{B}^{0}\left|\left(\frac{\partial^{2} V}{\partial Q_{a} \partial Q_{b}}\right)\right| \Psi_{A}^{0}\right\rangle=0
$$
stant, $K$

The totally symmetric component of $e_{2} \otimes e_{2}$ representation yields the harmonic force field con-

$$
K=\left\langle\Psi_{A}^{0}\left|\left(\frac{\partial^{2} V}{\partial Q_{a}^{2}}\right)\right| \Psi_{B}^{0}\right\rangle
$$

Using the Wiegner-Eckart theorem [30,31] and the coupling coefficients for the $D_{5}$ point group (Table 1), it is easy to see that the following integrals of eq. 4 vanish:

$$
\left\langle\Psi_{A}^{0}\left|\left(\frac{\partial^{V}}{\partial Q_{a}}\right)\right| \Psi_{B}^{0}\right\rangle=\left\langle\Psi_{B}^{0}\left|\left(\frac{\partial^{V}}{\partial Q_{a}}\right)\right| \Psi_{A}^{0}\right\rangle=\left\langle\Psi_{A}^{0}\left|\left(\frac{\partial^{V}}{\partial Q_{b}}\right)\right| \Psi_{A}^{0}\right\rangle=\left\langle\Psi_{B}^{0}\left|\left(\frac{\partial^{V}}{\partial Q_{b}}\right)\right| \Psi_{B}^{0}\right\rangle=0
$$


and the remaining integrals are

$$
\left.F=\left\langle\Psi_{A}^{0}\left|\left(\frac{\partial^{V}}{\partial Q_{a}}\right)\right| \Psi_{A}^{0}\right\rangle=-\left\langle\Psi_{B}^{0}\left|\left(\frac{\partial^{V}}{\partial Q_{a}}\right)\right| \Psi_{B}^{0}\right\rangle=-\left\langle\Psi_{A}^{0}\left|\left(\frac{\partial^{V}}{\partial Q_{b}}\right)\right| \Psi_{B}^{0}\right\rangle=-\left\langle\Psi_{B}^{0}\right| \frac{\partial^{V}}{\partial Q_{b}}\right)\left|\Psi_{A}^{0}\right\rangle
$$

Table 1 Coupling coefficients for the $D_{5}$ group (MATLAB scripts for the calculation of the coupling coefficients can be obtained from the authors).

\begin{tabular}{lclccc}
\hline Vibration & $\begin{array}{c}\text { El. state } \\
\text { component }\end{array}$ & \multicolumn{1}{c}{$\mathrm{AA}$} & \multicolumn{4}{c}{$E_{1} \times E_{1}$} & $\mathrm{AB}$ & $\mathrm{BB}$ \\
\hline$e_{2}$ & $b$ & 0 & -0.7071 & -0.7071 & 0 \\
& $a$ & 0.7071 & 0 & 0 & -0.7071 \\
\hline
\end{tabular}

The potential energy surfaces are thus

$$
\begin{aligned}
& E_{1}=E^{0}+\frac{1}{2} K\left(Q_{a}^{2}+Q_{b}^{2}\right)+F\left[\left(Q_{a}^{2}+Q_{b}^{2}\right)\right]^{1 / 2} \\
& E_{2}=E^{0}+\frac{1}{2} K\left(Q_{a}^{2}+Q_{b}^{2}\right)+F\left[\left(Q_{a}^{2}+Q_{b}^{2}\right)\right]^{1 / 2}
\end{aligned}
$$

From expression 10, it is easy to see that the energy along $Q_{a}$, or $Q_{b}$ or along any linear combination is the same. In this expression (eq. 10), only quadratic forms of $Q_{a}$ and $Q_{b}$ are present, thus the energy of a distortion along $-Q_{a}$ is the same energy as along $Q_{a}$, thus only the other component of the degenerate state will be stabilized. The potential energy surface has a "Mexican hat" shape, without any warping. Energy is the same in all directions of the 2D space spanned by these two coordinates.

As a result, the vibronic model employed in this study is defined with two parameters only, i.e., the linear vibronic coupling constant, $F$, and the force constant $K$.

\section{DFT CALCULATION OF THE JAHN-TELLER GROUND-STATE PROPERTIES}

Vibronic coupling constants mentioned in previous sections define the JT potential energy surface. A qualitative cross-section through the potential energy surface, along JT active vibration $Q_{a}$ is given in Fig. 2. The figure indicates also how the parameters $E_{\mathrm{JT}}$ (the JT stabilization energy), $\Delta$ (the warping barrier), $R_{\mathrm{JT}}$ (the JT radius), and $E_{\mathrm{FC}}$ (the Frank-Condon transition) define the potential energy surface. 


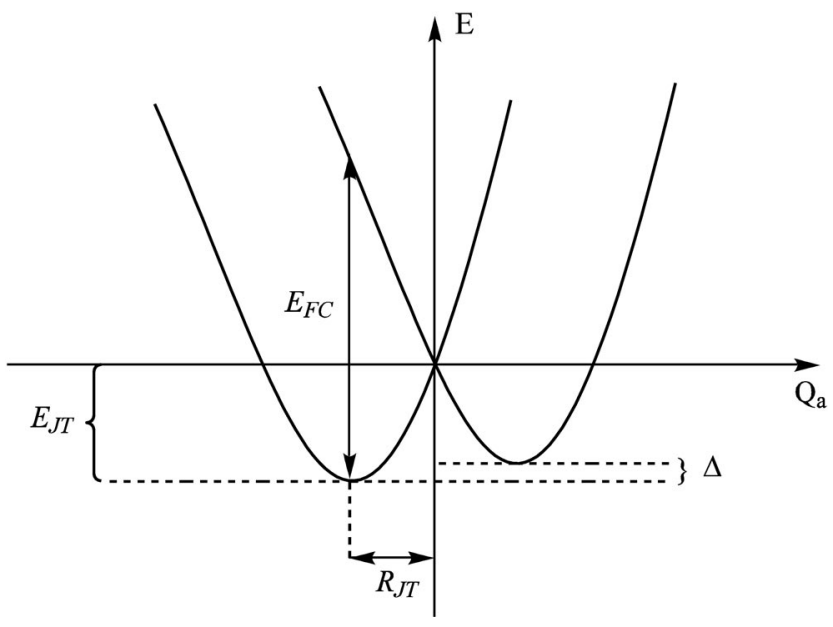

Fig. 2 Qualitative cross-section through the potential energy surface along the JT active coordinate $Q_{a}$; definition of the JT parameters - the JT stabilization energy $E_{\mathrm{JT}}$, the warping barrier $\Delta$, the JT radius $R_{\mathrm{JT}}$, the energy of the vertical Franck-Condon transition $E_{\mathrm{FC}}$.

Using first principles [11,34], it is possible to calculate $E_{\mathrm{JT}}, \Delta$, the geometries at the high-symmetry point and the global minimum, as well as the distance between them $\left(R_{\mathrm{JT}}\right)$. These alternative parameters are connected to the set of parameters discussed in previous sections using the following relations [2]:

$$
\begin{aligned}
& E_{\mathrm{JT}}=\frac{F^{2}}{2(K-2|G|)}=\frac{F^{2}}{2 K} \\
& \Delta=\frac{4 E_{\mathrm{JT}}|G|}{K+2|G|} \\
& R_{\mathrm{JT}}^{\min }=\frac{|F|}{K-2|G|}=\frac{F}{K}=-\frac{|F|}{K+2|G|}=R_{\mathrm{JT}}^{\mathrm{TS}}
\end{aligned}
$$

In this paper, we use DFT, today the method of choice in theoretical coordination chemistry. DFT is a single-determinant method. Therefore, it is not possible to derive correct energies in the case of orbital degeneracy [11]. In a non-empirical approach to ligand field theory [11], it was proposed to place 0.5 electrons into each of the e orbitals leading to a homogeneous distribution of electrons with partial occupations. This yields the geometry of the high-symmetry species. The JT stabilization energy is not simply the energy difference between the former high-symmetry and the low-symmetry species. To obtain $E_{\mathrm{JT}}$, we need two types of DFT calculations: (i) a single-point calculation imposing the high symmetry on the nuclear geometry and the low symmetry on the electron density. This gives the energy of a Slater determinant with a one-electron orbital occupancy. (ii) A geometry optimization in the lower symmetry. The $E_{\mathrm{JT}}$ is the difference in these two energies.

In order to discuss the JT potential surface, we define a distortion vector, $\vec{d}$ as the vector given by the displacements of the atoms in mass-weighted coordinates from the high-symmetry point defined by the $\vec{R}_{H}$. The difference between the high-symmetry geometry and the minimum geometry in massweighted coordinates allows us a definition of the $\mathrm{JT}$ radius, $R_{\mathrm{JT}}$, as a length of the corresponding vector $\left(\vec{d}_{J T}^{u}\right.$ being an unit vector), c.f. eq. 14 : 


$$
\vec{d}_{\mathrm{JT}}=\vec{D}_{5}-\vec{C}_{2}^{\min }=R_{\mathrm{JT}} \vec{d}_{\mathrm{JT}}^{u}
$$

\section{Computational details}

The DFT calculations reported in this work have been carried out using the ADF2006.01 program package [35-37]. The local density approximation (LDA) characterized by the Vosko-Willk-Nusair (VWN) [38] parameterization have been used for geometry optimizations. Triple-zeta (TZP) Slater-type orbital (STO) basis set have been used for all atoms. All calculations were spin-unrestricted with strict criteria for convergence: energy $10^{-4}$ Hartrees; gradients $10^{-4}$ Hartree/Å; changes in Cartesian coordinates $10^{-4} \AA$; and for numerical integration, 10 significant digits are used. For the energies, LDA and the generalized gradient approximation (GGA) in the form given by Perdew-Wang (PW91) [39,40] have been compared. Analytical harmonic frequencies were calculated [41-43] and analyzed with the aid of PyVib2 1.1 [44,45]. For the detailed discussion of potential energy surface, the IRC method [22,23] as implemented in the Amsterdam density functional (ADF) $[24,25]$ has been used. The initial direction of the path is chosen by computing the gradient at the high-symmetry configuration.

\section{RESULTS AND DISCUSSION}

As indicated above, DFT produces a totally symmetric electron distribution $\left(\rho_{0}\right)$ if each $\mathrm{e}_{1}$ orbital carries 0.5 electrons. There are two distinct ways to accommodate the single electron in $C_{2}$ symmetry, i.e., $\mathrm{a}^{1} \mathrm{~b}^{0}\left({ }^{2} \mathrm{~A}\right.$ electronic state) yielding a density $\rho(A)$ and a geometry of the energy minimum $C_{2}(A)$ or $\mathrm{b}^{1} \mathrm{a}^{0}$ $\left({ }^{2} \mathrm{~B}\right.$ electronic state) yielding another density $\rho(B)$ with a geometry of the energy minimum $C_{2}(B)$. The two electronic states are stabilized in opposite directions of $Q_{a}$. Thus, a series of DFT calculations corresponding to both of these occupations, as well as to the $G_{0}=D_{5}$ and $G_{1}=C_{2}$ geometries, are carried out, leading to the values of JT stabilization energies, $E_{\mathrm{JT}}(A)$ and $E_{\mathrm{JT}}(B)$. The energy of vertical Franck-Condon transition, $E_{\mathrm{FC}}$, is easily obtained in promoting the unpaired electron from the ground state to the first excited state for the ground-state geometry. For the definition of the parameters, see Fig. 2. Calculations were done for both eclipsed and staggered conformations.

Results of these calculations are tabulated in Table 2.

Table 2 Results of the DFT calculations performed to analyze the JT effect of cobaltocene in $D_{5 h}$ and $D_{5 d}$ symmetry; geometries are obtain with LDA; energies (LDA and GGA) are given in eV; symmetry symbols are for $D_{5}$ as explained in text.

\begin{tabular}{|c|c|c|c|c|c|c|}
\hline \multirow[b]{2}{*}{ Occupation } & \multirow[b]{2}{*}{ State } & \multirow[b]{2}{*}{ Geometry } & \multicolumn{2}{|c|}{ Eclipsed } & \multicolumn{2}{|c|}{ Staggered } \\
\hline & & & $E(\mathrm{LDA})$ & $E(\mathrm{GGA})$ & $E(\mathrm{LDA})$ & $E(\mathrm{GGA})$ \\
\hline $\mathrm{e}_{1}^{0.5} \mathrm{e}_{1}^{0.5}$ & ${ }^{2} \mathrm{E}_{1}$ & $D_{5}$ & -142.28971 & -132.95708 & -142.26204 & -132.93751 \\
\hline$a^{1} b^{0}$ & ${ }^{2} \mathrm{~A}$ & $D_{5}$ & -142.26105 & -132.97115 & -142.23739 & -132.95772 \\
\hline$a^{1} b^{0}$ & ${ }^{2} \mathrm{~A}$ & $C_{2}(A)$ & -142.36200 & -133.06415 & -142.33129 & -133.04308 \\
\hline$a^{1} b^{0}$ & ${ }^{2} \mathrm{~B}$ & $C_{2}(A)$ & -141.95712 & -132.65895 & -141.93846 & -132.65035 \\
\hline$b^{1} a^{0}$ & ${ }^{2} \mathrm{~B}$ & $D_{5}$ & -142.26113 & -132.97103 & -142.23740 & -132.95769 \\
\hline$b^{1} a^{0}$ & ${ }^{2} \mathrm{~B}$ & $C_{2}(B)$ & -142.36199 & -133.06395 & -142.33127 & -133.04316 \\
\hline$b^{0} a^{1}$ & ${ }^{2} \mathrm{~A}$ & $C_{2}(B)$ & -141.95678 & -132.65842 & -141.93882 & -132.65076 \\
\hline
\end{tabular}

Cobaltocene in $D_{5 h}$ symmetry is approximately $0.02-0.03 \mathrm{eV}$ more stable than in $D_{5 d}$. The energy difference between the low-symmetry conformations $C_{2 v}$ and $C_{2 h}$ obtained by descent in symmetry from $D_{5 h}$ and $D_{5 d}$ is similar. This is in agreement with results of previous DFT calculations on metallocenes [46,47]. The energy barrier for the internal rotation of the rings, from eclipsed to staggered conformation, is estimated to be around $0.03 \mathrm{eV}$ in both high and low symmetries, similar to the rotation of the rings in ferrocene [48]. 
The JT parameters are easily obtained from these results, cf. Table 3.

Table 3 The JT parameters; energies are given in $\mathrm{cm}^{-1} ; R_{\mathrm{JT}}$ in $(\mathrm{amu})^{1 / 2} \AA ; F$, in $10^{3} \mathrm{~cm}^{-1}(\mathrm{amu})^{-1 / 2} \AA^{-1} ; K$, in $10^{3} \mathrm{~cm}^{-1}$ $(\mathrm{amu}) \AA ̊$.

\begin{tabular}{lcrrr}
\hline & \multicolumn{2}{c}{$D_{5 h}$} & \multicolumn{2}{c}{$D_{5 d}$} \\
& LDA & GGA & LDA & GGA \\
\hline$E_{\mathrm{JT}}(A)$ & 814.2 & 750.1 & 757.4 & 688.5 \\
$E_{\mathrm{JT}}(B)$ & 813.5 & 749.5 & 757.1 & 689.4 \\
$\Delta$ & 0.7 & 0.6 & 0.3 & 0.9 \\
$E_{\mathrm{FC}}(A)$ & 3265.6 & 3268.2 & 3168.4 & 3167.6 \\
$E_{\mathrm{FC}}(B)$ & 3268.3 & 3270.8 & 3165.3 & 3164.9 \\
$R_{\mathrm{JT}}(A)$ & 0.35 & & 0.34 & \\
$R_{\mathrm{JT}}(B)$ & 0.35 & & 0.34 & \\
$F$ & 4.6 & 4.3 & 4.4 & 4.0 \\
$K$ & 13.3 & 12.2 & 13.1 & 11.9 \\
\hline
\end{tabular}

JT stabilization energies are in range $688.5-814.2 \mathrm{~cm}^{-1}$, depending on the structure and on the functional used for the energy calculation. These values are in good agreement with the value of $1050 \mathrm{~cm}^{-1}$ estimated from the experiment [15]. The JT stabilization energy is somewhat larger in the case of eclipsed rings. The ratio $E_{\mathrm{FC}} / E_{\mathrm{JT}}$ is almost exactly 4 as predicted by the model with LDA (4.01) and a little larger for GGA energies (4.35). The JT energies for the different electronic states are almost exactly the same-the differences are between 0.3 and $0.9 \mathrm{~cm}^{-1}$, smaller then the precision of the calculations. As expected, based on group theoretical considerations, there is no warping of the potential energy surface, and the vibronic model employed in this study is described with two parameters-only the linear vibronic coupling constant, $F$, and the force constant $K$, as previously explained, Table 3.

The geometrical parameters for all the structures studied in this work are summarized in Table 4. The cyclopentadienyl rings are not planar, and the aromaticity of the ring is perturbed. There are no significant structural changes of the rings in the low symmetry eclipsed $\left(C_{2 v}\right)$ as well as for the staggered $\left(C_{2 h}\right)$ conformations. This strongly suggests that the energy barrier for the rotation of the rings is as small as in ferrocene. This was verified by calculating the energy profile for the ring rotation. The JT distortion does not influence the internal rotation of the two rings. A detailed analysis of the geometrical deformation from the higher symmetry follows.

Table 4 Geometrical parameters for different structures calculated in this work, optimized with LDA; distances in $\AA$ and torsional angles in ${ }^{\circ}$; for the numbering of $\mathrm{C}$ atoms, see Fig. 1.

\begin{tabular}{lcccccc}
\hline & $D_{5 h}$ & $D_{5 d}$ & $C_{2 v}\left(A_{2}\right)$ & $C_{2 v}\left(B_{1}\right)$ & $C_{2 h}\left(A_{g}\right)$ & $C_{2 h}\left(B_{g}\right)$ \\
\hline $\mathrm{Co}_{-} \mathrm{C}_{1}$ & 2.068 & 2.071 & 2.028 & 2.104 & 2.107 & 2.033 \\
$\mathrm{Co}-\mathrm{C}_{2}\left(\mathrm{C}_{5}\right)$ & & & 2.097 & 2.035 & 2.039 & 2.098 \\
$\mathrm{Co}-\mathrm{C}_{3}\left(\mathrm{C}_{4}\right)$ & & & 2.054 & 2.077 & 2.078 & 2.056 \\
$\mathrm{C}_{1}-\mathrm{C}_{2}\left(\mathrm{C}_{1}-\mathrm{C}_{5}\right)$ & 1.421 & 1.420 & 1.427 & 1.416 & 1.416 & 1.427 \\
$\mathrm{C}_{2}-\mathrm{C}_{3}\left(\mathrm{C}_{4}-\mathrm{C}_{5}\right)$ & & & 1.407 & 1.436 & 1.435 & 1.406 \\
$\mathrm{C}_{3}-\mathrm{C}_{4}$ & & & 1.439 & 1.403 & 1.403 & 1.439 \\
$\Theta_{\mathrm{C} 1-\mathrm{C} 2}\left(-\Theta_{\mathrm{C} 4-\mathrm{C} 5}\right)$ & 0.0 & 0.0 & 6.4 & 6.4 & 6.2 & 6.2 \\
$\Theta_{\mathrm{C} 2-\mathrm{C} 3}\left(-\Theta_{\mathrm{C} 3-\mathrm{C} 4}\right)$ & & & 3.9 & 3.9 & 3.9 & 3.8 \\
$\Theta_{\mathrm{C} 5-\mathrm{C} 1}$ & & & 0.0 & 0.0 & 0.0 & 0.0 \\
\hline
\end{tabular}




\section{Analysis of JT distortion}

As shown in the previous sections, the JT theorem predicts a distortion from the high-symmetry configuration. By group theory, we find the irreducible representation of the JT active vibrations, and by using the epikernal principle $[2,32,33]$ we obtain the symmetry descent. The geometry of minimal energy defines the direction of the JT distortion. The JT radius is given by the length of the distortion vector between the high-symmetry and minimum-energy configuration.

The distortions of simple complexes are usually determined by one single normal coordinate that satisfies the symmetry requirements [2]. In complex molecules, the JT distortion is a superposition of different normal coordinates. A detailed analysis of the different contributions is of interest in this case.

The qualitative treatment shows that the JT distortion is defined in the subspace of the two components of the $e_{2}$ direction. The real situation, however, is more complicated. There are more than one $e_{2}$ vibrations, and because they are of the same symmetry they can all contribute to the JT distortion. Since $\mathrm{E}_{1} \otimes \mathrm{E}_{1} \subset a_{1}+\left[a_{2}\right]+\left[e_{2}\right]$ also $a_{1}$ modes may be effective in addition to the $e_{2}$ vibrations. Totally symmetric displacements do not change the symmetry, they change only the interatomic distances. Hence, all of the $e_{2}$ vibrations and all totally symmetric vibrations can contribute to the JT distortion. In the JT semantics, this is called the multimode problem [2]. Further complications can arise after the actual symmetry is lowered. Then, all vibrations which are totally symmetric in the lower symmetry can contribute to the JT distortion. Thus, in $C_{2 v}$ symmetry we have 16 totally symmetric vibrations and in $C_{2 h} 15$. Hence, the question arises what is their contribution to the JT distortion of a molecule. This is easily answered if we express JT active distortion as a linear combination of all totally symmetric normal modes in the low-symmetry $\left(C_{2}\right)$ minimum-energy conformation. This is the essence of our proposition how to treat the multimode problem. More details about this approach will be published shortly [49].

$$
\vec{d}=c_{1} \vec{q}_{1}\left(C_{2}\right)+c_{2} \vec{q}_{2}\left(C_{2}\right)+\cdots+c_{n} \vec{q}_{n}\left(C_{2}\right)=\sum_{i=1}^{n} c_{i} \vec{q}_{i}\left(C_{2}\right)
$$

The set of normal modes $\mathbf{q}=\left(\vec{q}_{1}, \vec{q}_{2}, \ldots, \vec{q}_{n}\right), n$ being the number of the totally symmetric vibrations in low symmetry, are orthonormal, that is, $\vec{q}_{i} \cdot \vec{q}_{j}=\delta_{i j}$ :

$$
\begin{aligned}
& \vec{q}_{i} \cdot \vec{d}=\sum_{i=1}^{n} c_{i} \delta_{i j} \\
& \mathbf{q} \cdot \vec{d}=\mathbf{c}=\left\{c_{1}, c_{2}, \cdots, c_{n}\right\}
\end{aligned}
$$

In order to be consistent with the usual treatments [2] and the previous qualitative arguments, we correlate the vibrations in the minimum-energy conformation of low symmetry to the ones in high symmetry by the method developed by Hug et al. [44,45], using overlap and similarities of the massweighted Cartesian displacements of the two different configurations.

Normal coordinate analysis at the minima yields the energies of the normal modes and the corresponding Cartesian displacements. The $c_{i} \mathrm{~s}$, which are normalized to 1 , thus represent the percentage of any particular normal mode to the total JT distortion and are obtained using eq. 17 . With this information, it is possible to estimate the energy contribution of each of the $n$ vibrations to $E_{\mathrm{JT}}$. The results corresponding to the ${ }^{2} \mathrm{~A}_{2}$ ground state in $C_{2 v}$ geometry are given in Table 5. Similar results are obtained for the companion state ${ }^{2} \mathrm{~B}_{1}$, as well as for the corresponding states in $C_{2 h}$ symmetry. These results are given in the Supplementary Information. Assignment of the vibrations is given according to the normal coordinate analysis of the ferrocene and ruthenocene [48,50-52]. 
Table 5 Linear coefficients, $c_{i}$, in the expansion of the distortion vector, normalized to 1 , at the minimum of ${ }^{2} \mathrm{~A}_{2}$ electronic state in $C_{2 v}$ geometry; correlation of the low- to the high-symmetry vibrations is given; contribution of the normal coordinate to the calculated $E_{\mathrm{JT}}$ in $\mathrm{cm}^{-1}$. The most important vibrations are given in bold; energy of vibrations in $\mathrm{cm}^{-1}$ and their assignment is given [44-49].

\begin{tabular}{|c|c|c|c|c|c|}
\hline & $\begin{array}{l}\text { Energy of the vib. } \\
\text { in } C_{2 v}\left(\mathrm{~cm}^{-1}\right)\end{array}$ & Assigment of the vib. & $\begin{array}{l}\text { Symmetry of the } \\
\text { vib. in } D_{5 h}\end{array}$ & $c_{i}\left(R=R_{\mathrm{JT}}\right)$ & $E_{\mathrm{JT}}^{i}\left(\mathrm{~cm}^{-1}\right)$ \\
\hline 1 & 153.2 & skeletal bending & $e_{1}^{\prime}$ & 0.0004 & 0.0121 \\
\hline 2 & 291.9 & ring-metal stretch & $a_{1}^{\prime}$ & 0.0026 & 1.0562 \\
\hline 3 & 405.5 & ring tilt & $e_{1}^{\prime}$ & 0.0021 & 0.6525 \\
\hline 4 & 586.9 & out-of-plane ring deformation & $e_{2}^{\prime}$ & 0.6490 & 348.1601 \\
\hline 5 & 762.0 & $\mathrm{C}-\mathrm{H}$ wagging & $a_{1}^{\prime}$ & 0.0041 & 5.2601 \\
\hline 6 & 825.4 & $\mathrm{C}-\mathrm{H}$ wagging & $e_{1}^{\prime}$ & 0.0138 & 9.4111 \\
\hline 7 & 830.3 & in-plane ring distortion & $e_{2}^{\prime}$ & 0.1004 & 112.0479 \\
\hline 8 & 869.1 & C-H wagging & $e_{2}^{\prime}$ & 0.1216 & 137.0990 \\
\hline 9 & 976.0 & $\mathrm{C}-\mathrm{H}$ bending & $e_{1}^{\prime}$ & 0.0009 & 2.7961 \\
\hline 10 & 1030.8 & in-plane $\mathrm{C}-\mathrm{H}$ bending & $e_{2}^{\prime}$ & 0.0833 & 133.7086 \\
\hline 11 & 1126.4 & ring breathing mode ( $\mathrm{C}-\mathrm{C}$ stretch) & $a_{1}{ }^{\prime}$ & 0.0004 & 0.0012 \\
\hline 12 & 1367.6 & $\mathrm{C}-\mathrm{C}$ stretch & $e_{2}^{\prime}$ & 0.0098 & 34.2503 \\
\hline 13 & 1397.6 & $\mathrm{C}-\mathrm{C}$ stretch & $e_{1}^{\prime}$ & 0.0118 & 28.1925 \\
\hline 14 & 3135.9 & C-H stretch & $e_{2}^{\prime}$ & 0.0004 & 1.1785 \\
\hline 15 & 3148.3 & C-H stretch & $e_{1}^{\prime}$ & 0.0000 & 0.3532 \\
\hline 16 & 3166.2 & $\mathrm{C}-\mathrm{H}$ stretch & $a_{1}^{\prime}$ & 0.0000 & 0.0205 \\
\hline
\end{tabular}

The mixing of the totally symmetric vibrations in lower symmetry is expected to increase with increasing deviation from the high-symmetry geometry. It is interesting to see how the composition of distortion vector (coefficients in the linear expansion, eq. 15) changes along the minimal energy path. The later is defined as the steepest descent path [22,23], down from the JT cusp to the local energy minimum. This is easily achieved using the IRC algorithm as implemented by Deng and Ziegler [24,25] in the ADF program package [35-37]. The high-symmetry point has a nonzero gradient, thus, the first step is computed in the direction of the steepest descent (not in the direction of the first Hessian eigenvector as usually in IRC calculations starting from the transition states). The path is computed by taking steps of adequate size and by optimizing all atomic coordinates orthogonal to it. During the calculation, $C_{2 v}$ symmetry has been conserved, and it is taken into account that one electronic state corresponds to the forward path, and the other to the backward path. Each point along the minimal energy path can be characterized by the distortion vector $\vec{d}$. As already demonstrated, it represents the difference between the high-symmetry and current point, and it can be represented as the linear combination of the totally symmetric normal modes of the low-symmetry minimum conformation

$$
\begin{aligned}
& \vec{d}=\vec{D}_{5}-\vec{C}_{2}=R \vec{d}^{u} \\
& \vec{d}=\sum_{i=1}^{n} c_{i} \vec{q}_{i}\left(C_{2}\right)
\end{aligned}
$$

IRC calculations for the eclipsed conformation of the rings are summarized in Fig. 3 together with the direct path. It can be seen that these two ways are not significantly different. 


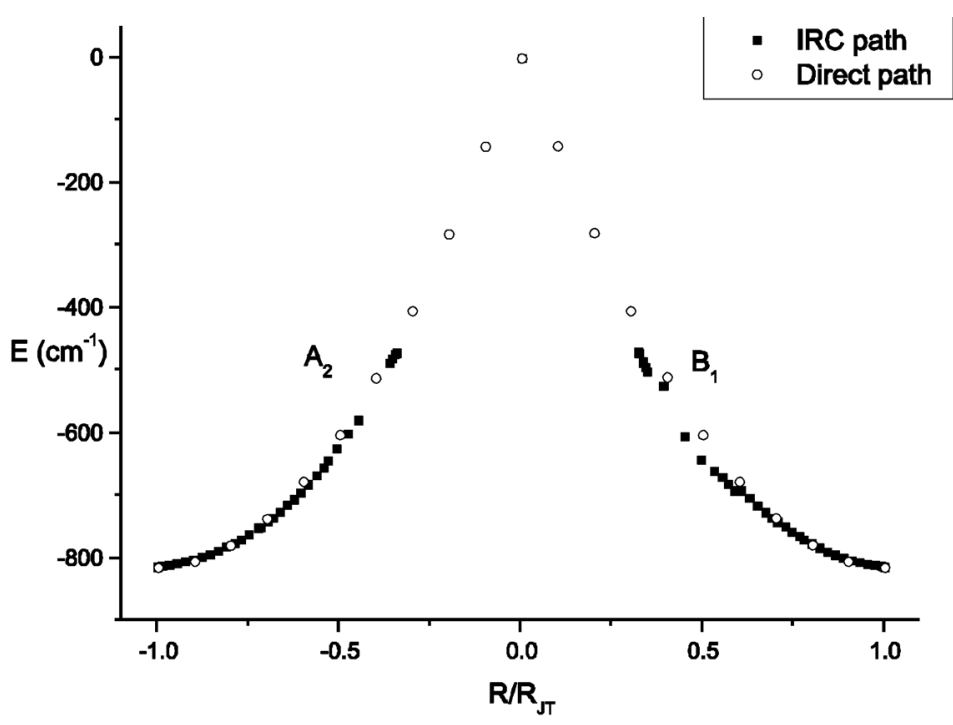

Fig. 3 IRC calculations (filled squares) and direct path (open circles) from the high-symmetry cusp in $C_{2 v}$ symmetry (eclipsed conformation of the rings); forward direction is ${ }^{2} \mathrm{~B}_{1}$ electronic state; backward direction is ${ }^{2} \mathrm{~A}_{2}$ electronic state; energies are given in $\mathrm{cm}^{-1}$ relative to the high-symmetry one.

Changes of $c_{i}$ for the four dominant vibrations along the IRC path are represented in Fig. 4.

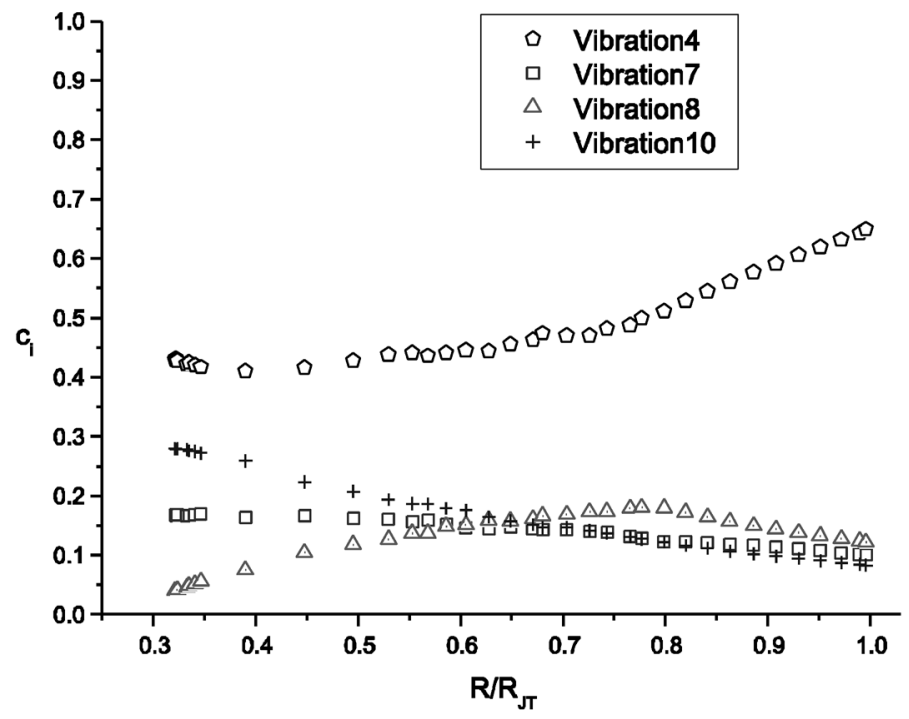

Fig. 4 Changes in the composition of the distortion vector $\vec{d}=\vec{D}_{5}-\vec{C}_{2}$ (linear coefficients, $c_{i}$ in eq. 15) along the minimal energy (IRC) path for the four most important vibrations. Linear coefficients are normalized to 1.

The main contribution to the JT distortion arises from the four $e_{2}$ type vibrations (labeled as 4,7 , 8 , and 10 in Table 5). They contribute to about $95 \%$ of the total JT distortion vector and to $90 \%$ of the JT energy. The four vibrations are: the out-of-plane ring distortion, 4, the in-plane ring distortion, 7, the $\mathrm{C}-\mathrm{H}$ wagging (the out-of-plane $\mathrm{C}-\mathrm{H}$ bending), 8, and the in-plane $\mathrm{C}-\mathrm{H}$ bending, 10. 
These vibrations are illustrated in Fig. 5 using the vibrational energy distribution representation [40]. The different colors indicate the direction of the displacement vector, while the volumes of the spheres are proportional to the contribution made by the individual nuclei to the energy of the vibrational mode. Their significance is not the same at the beginning step and at the minimum. Figure 4 shows that the composition of the distortion vector changes along the minimal energy path. For infinitesimal small distortion from the high symmetry, we expect only contribution of $e_{2}$ vibrations. The analysis shows that the contribution of low-energy skeletal vibrations (1-3) and the high-energy vibrations (C-C stretch 11-13, and $\mathrm{C}-\mathrm{H}$ stretch $14-16)$ is almost negligible. The JT important $e_{2}$ vibrations, and hence the JT distortion, are predominantly located in the five-membered rings. The main contribution is the out-of-plane deformation of cyclopentadienyl ring (vibration 4). This is expected because this normal coordinate minimizes antibonding interactions between the cyclopentadienil ring orbitals and the single occupied metal d orbital. In the beginning, the contribution of in-plane $\mathrm{C}-\mathrm{H}$ bending, 10 , is also important, but as distortion deviates from the high-symmetry point its contribution decreases. The opposite is true for the lowest energy $e_{2}$ vibration (vibration 4) which is indeed the most important one. Contribution of $\mathrm{C}-\mathrm{H}$ wagging is also becoming more important. On the first sight, it might be surprising that the softest of the four modes makes the largest contribution to $E_{\mathrm{JT}}$. This indicates that the distortion along the corresponding normal coordinate is larger than for any other one.

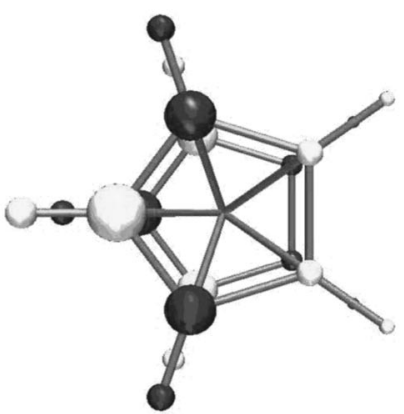

4. out-of-plane ring deformation $568.9 \mathrm{~cm}^{-1}$

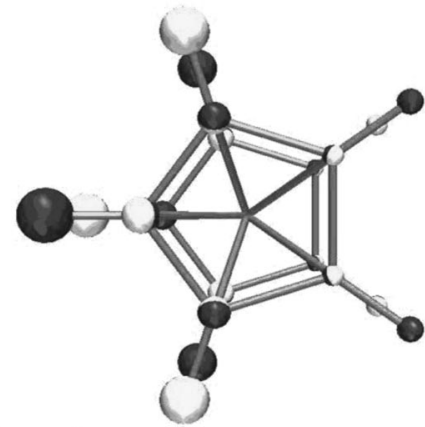

8. C-H wagging

$869.1 \mathrm{~cm}^{-1}$

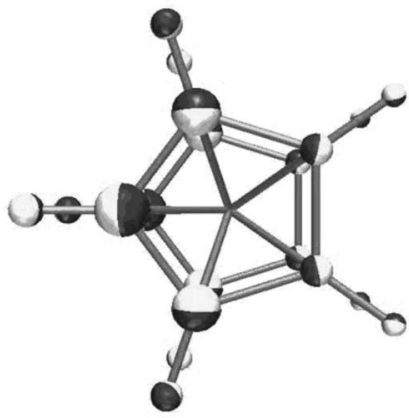

7. in-plane ring deformation $830.3 \mathrm{~cm}^{-1}$

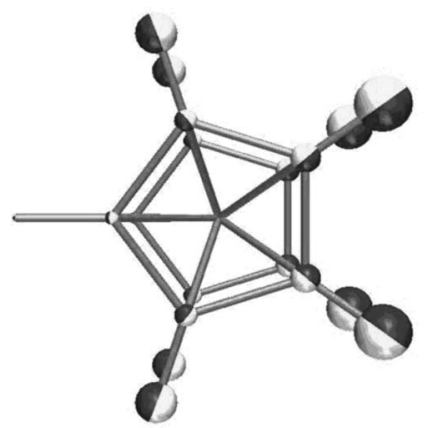

10. in-plane $\mathrm{C}-\mathrm{H}$ bending $1030.8 \mathrm{~cm}^{-1}$

Fig. 5 Vibrational energy distribution representation of the four most important $a$ vibrations in $C_{2 v}$ symmetry corresponding to the four $e_{2}^{\prime}$ vibrations in $D_{5 h}$ symmetry (Table 5); upper row: vibration 4 (out-of-plane ring deformation) and vibration 7 (in-plane ring distortion); down: vibration 8 (C-H wagging) and vibration 10 (inplane $\mathrm{C}-\mathrm{H}$ bending). The different colors indicate the direction of the displacement vector; the volume of the spheres is proportional to the contribution made by the individual nuclei to the energy of the vibrational mode. 


\section{CONCLUSIONS}

In this work, we have shown that methods based on DFT are useful tools for the qualitative and quantitative analysis of the adiabatic potential energy surfaces of JT active coordination compounds. The composition of the distortion vector that describes the displacements of all atoms allows the discussion of the direction and of the magnitude (through the JT radius) of the JT effect. The JT distortion is a totally symmetric reaction coordinate in low symmetry and can be expressed as a linear combination of all normal modes of $a_{1}$ irreps. in the low-symmetry energy minimum. These vibrations can be correlated to the vibrations in the high-symmetry configuration, thus making the bridge between this approach and the standard one based on the perturbation of the high-symmetry conformation. Using points along the minimal energy path, it is possible to follow the changes of composition of the distortion vector from the high-symmetry structure to the energy minimum.

In the particular example of the cobaltocene molecule, the three vibronic coupling constants have been calculated. The quadratic one, $G_{A B}^{a b}$, is found to be zero as predicted by group theory. Four $e_{2}$ type deformation modes in high symmetry, the $a$ component after descent in symmetry, are identified as the most important contribution to the JT deformation, with the out-of-plane ring deformation being dominant.

\section{SUPPLEMENTARY INFORMATION}

Tables S1-S4 are available online (doi:10.1351/PAC-CON-08-06-04).

\section{ACKNOWLEDGMENTS}

This work was supported by the Swiss National Science Foundation. MZ thanks to the Serbian Ministry of Science and Environmental Protection (Grant No. 142017G) and to the Federal Commission for Scholarships for Foreign Students of the Swiss Government (ESKAS) for a scholarship.

\section{REFERENCES}

1. H. A. Jahn, E. Teller. Proc. R. Soc. London, Ser. A 161, 220 (1937).

2. I. B. Bersuker. The Jahn-Teller Effect, Cambridge University Press (2006).

3. R. G. Pearson. Symmetry Rules for Chemical Reactions, Wiley-Interscience, New York (1976).

4. A. D. Liehr. J. Phys. Chem. 67, 389 (1963).

5. R. Boca, M. Breza, P. Pelikan. Struct. Bonding 71, 57 (1989).

6. D. Reinen, M. Atanasov. Magn. Reson. Rev. 15, 167 (1991).

7. I. B. Bersuker. J. Comput. Chem. 18, 260 (1997).

8. R. G. Parr, W. Yang. Density-Functional Theory of Atoms and Molecules, Oxford University Press (1989).

9. T. Ziegler, A. Rauk, E. J. Baerends. Theor. Chim. Acta 43, 261 (1977).

10. C. Daul. Int. J. Quant. Chem. 52, 867 (1994).

11. R. Bruyndockx, C. Daul, P. T. Manoharan, E. Deiss. Inorg. Chem. 36, 4251 (1997).

12. T. K. Kundu, R. Bruyndockx, C. Daul, P. T. Manoharan. Inorg. Chem. 38, 3931 (1999).

13. M. Atanasov, P. Comba, C. Daul, A. Hauser. J. Phys. Chem. A 111, 9145 (2007).

14. J. H. Ammeter, J. D. Swallen. J. Chem. Phys. 57, 678 (1972).

15. R. Bucher. ESR-Untersuchungen an Jahn-Teller-Aktiven Sandwitchkomplexen, Ph.D. thesis, ETH Zürich (1977).

16. E. Köning, R. Schnakig, S. Kremer, B. Kanellakopulos, R. Klenze. Chem. Phys. 27, 331 (1978).

17. J. H. Ammeter, L. Zoller, J. Bachmann, P. Baltzer, E. Gamp, R. Bucher, E. Deiss. Helv. Chim. Acta 64, 1063 (1981). 
18. J. Weber, A. Goursot, E. Pénigault, J. H. Ammeter, J. Bachmann. J. Am. Chem Soc. 104, 1491 (1982).

19. A. Stebler, A. Furrer, J. H. Ammeter. Inorg. Chem. 23, 3493 (1984).

20. H. Eicher. Phys. Rev. A 40, 1637 (1989).

21. N. Matsuzawa, J. Seto, D. Dixon. J. Phys. Chem. A 101, 9391 (1997).

22. K. Fukui. J. Phys. Chem. 74, 4161 (1970).

23. K. Fukui. Acc. Chem. Res. 14, 363 (1981).

24. L. Deng, T. Ziegler. J. Chem. Phys. 99, 3823 (1993).

25. L. Deng, T. Ziegler. Int. J. Quant. Chem. 52, 731 (1994).

26. R. Renner. Z. Phys. 92, 172 (1934).

27. R. F. W. Bader. Mol. Phys. 137, 3 (1960).

28. R. F. W. Bader. Can. J. Chem. 40, 1164 (1962).

29. R. F. W. Bader, A. D. Bandrauk. J. Chem. Phys. 49, 1666 (1968).

30. E. P. Wigner. Gruppentheorie, Vieweg, Braunschweig (1930).

31. C. Eckart. Rev. Mod. Phys. 2, 305 (1930).

32. A. Ceulemans, D. Beyens, L. G. Vanquickenborne. J. Am. Chem. Soc. 106, 5824 (1984).

33. A. Ceulemans, L. G. Vanquickenborne. Struct. Bonding 71, 125 (1989).

34. T. A. Barckholtz, T. A. Miller. J. Phys. Chem. A 103, 2321 (1999).

35. ADF2006.01, SCM, Theoretical Chemistry, Vrije Universiteit Amsterdam, The Netherlands, $<$ http://www.scm.com>.

36. G. te Velde, F. M. Bickelhaupt, S. J. A. Gisbergen, C. F. Guerra, E. J. Baerends, J. G. Snijders, T. Ziegler. J. Comput. Chem. 22, 931 (2001).

37. C. F. Guerra, J. G. Snijders, G. te Velde, E. J. Baerends. Theor. Chem. Acc. 99, 391 (1998).

38. S. Vosko, L. Wilk, M. Nusair. Can. J. Phys. 58, 1200 (1980).

39. J. Perdew, Y. Wang. Phys. Rev. B 33, 8822 (1986).

40. J. Perdew, J. Chevary, S. Vosko, K. Jackson, M. Pederson, D. Singh, C. Fiolhais. Phys. Rev. B 46, 6671 (1992).

41. A. Brces, R. M. Dickson, L. Fan, H. Jacobsen, D. Swerhone, T. Ziegler. Comput. Phys. Commun. 100, 247 (1997).

42. H. Jacobsen, A. Brces, D. Swerhone, T. Ziegler. Comput. Phys. Commun. 100, 263 (1997).

43. S. K. Wolff. Int. J. Quant. Chem. 104, 645 (2005).

44. W. Hug, M. Fedorovsky. Theor. Chem. Acc. 119, 113 (2008).

45. M. Fedorovsky. Comp. Lett. 2, 233 (2006).

46. Z.-F. Xu, Y. Xie, W.-L. Feng, H. F. Schaefer III. J. Phys. Chem. A 107, 2176 (1997).

47. M. Swart. Inorg. Chim. Acta 360, 179 (2007).

48. A. Bérces, T. Ziegler. Top. Curr Chem. 182, 41 (1996).

49. M. Zlatar, C.-W. Schlaepfer, C. Daul. In The Jahn-Teller Effect, Advances and Perspectives, H. Köppel, H. Barentzen, D. R. Yarkony (Eds.), Springer-Verlag (2009). In press.

50. J. Brunvoll, S. J. Cyvin, L. Schäfer. J. Organomet. Chem. 27, 107 (1971).

51. L. Schäfer, J. Brunvoll, S. J. Cyvin. J. Mol. Struct. 11, 459 (1972).

52. D. M. Adams, W. S. Fernando. J. Chem. Soc., Dalton Trans. 2507 (1972). 